\title{
Application of the $k$ - nearest neighbors algorithm for predicting blast - induced ground vibration in open - pit coal mines: a case study
}

\author{
Hoang Nguyen $1,2, *$ \\ 1 Department of Surface Mining, Mining Faculty, Hanoi University of Mining and Geology, Vietnam \\ ${ }^{2}$ Center for Mining, Electro - Mechanical Research, Hanoi University of Mining and Geology, Vietnam
}

\author{
ARTICLE INFO \\ Article history: \\ Received 15 ${ }^{\text {st }}$ Aug. 2020 \\ Revised 05 ${ }^{\text {th }}$ Dec. 2020 \\ Accepted 31 ${ }^{\text {st }}$ Dec. 2020 \\ Keywords: \\ Artificial intelligence, \\ Ground vibration, \\ $\mathrm{K}$ - nearest neighbors, \\ Machine learning, \\ Peak particle velocity.
}

\section{ABSTRACT}

Blasting is considered as one of the most effective methods for rock fragmentation in open - pit mines. However, its side effects are significant, especially blast - induced ground vibration. Therefore, this study aims to develop and apply artificial intelligence in predicting blast - induced ground vibration in open - pit mines. Indeed, the $k$ - nearest neighbors (KNN) algorithm was taken into account and developed for predicting blast - induced ground vibration at the Deo Nai open - pit coal mine (Vietnam) as a case study. An empirical model (i.e., USBM) was also developed to compare with the developed KNN model aiming to highlight the advantage of the KNN model. Accordingly, 194 blasting events were collected and analyzed for this aim. This database was then divided into two parts, $80 \%$ for training and 20\% for testing. The MinMax scale and 10 - fold cross - validation techniques were applied to improve the accuracy, as well as avoid overfitting of the KNN model. Root - mean squared error (RMSE) and determination coefficient $\left(R^{2}\right)$ were used as the performance metrics for models' evaluation and comparison purposes. The results indicated that the KNN model yielded better superior performance than those of the USBM empirical model with an RMSE of 1.157 and $R^{2}$ of 0.967. In contrast, the USBM model only provided a weak performance with an RMSE of 4.205 and $R^{2}$ of 0.416. With the obtained results, the KNN can be introduced as a potential artificial intelligence model for predicting and controlling blast - induced ground vibration in practical engineering, especially at the Deo Nai open - pit coal mine.
${ }^{*}$ Corresponding author

E - mail: nguyenhoang@humg.edu.vn DOI: 10.46326/JMES.2020.61(6).03

\section{Introduction}

Blasting is one of the most common methods for rock fragmentation in open - pit mines since its advantages in terms of economic and technical (Nguyen, 2019). However, according to scientists, 
it is not the entire of the explosive energy that is useful for rock fragmentation. Only $25 \div 30 \%$ of the total explosive energy was used for this aim, and the remaining energy is wasted (Hasanipanah et al., 2017). It generated undesirable effects, such as ground vibration, air over - pressure, fly - rock, back - break, and air pollution (Monjezi et al., 2010; Khandelwal, 2011; Armaghani et al., 2018; Fang et al., 2019a; Nguyen and Bui, 2019; Nguyen et al., 2020). Of those, blast - induced ground vibration is considered as the most hazardous phenomenon. It can make the vibration of buildings, bench/slope instability, and make discomposure for the residential (Bui et al., 2019; 2020). Therefore, accurate prediction of blast induced ground vibration is one of the efforts of researchers and engineers aiming to reduce the side effects on the surrounding environment.

In order to evaluate the intensity of blast induced ground vibration, peak particle velocity (PPV) is often used as a critical parameter in blasting operations. It can be estimated by empirical equations or artificial intelligence models (Armaghani et al., 2015; Ding et al., 2019; Fang et al., 2019a; Fang et al., 2019b; Nguyen et al., 2019a). Indeed, in recent years, AI techniques have been widely applied in predicting PPV. Many researchers proposed and applied different AI techniques for this aim. Monjezi et al., (2013) developed an artificial neural network (ANN) to predict PPV with a promising result. In another study, Armaghani et al., (2014) developed a hybrid model based on ANN and an optimization algorithm (i.e., particle swarm optimization - PSO) for predicting PPV, called PSO - ANN model. Their results are positive, and the PSO - ANN model was proposed as a potential model in blasting operations. In another study, they applied the imperialist competitive algorithm (ICA) for predicting PPV, and the positive results were reported as well (Armaghani et al., 2018). In another study, Ding et al., (2019) proposed a novel hybrid model, namely ICA - XGBoost, for predicting PPV. They claimed that this model could predict PPV with high accuracy. Hajihassani et al., (2015) also proposed a potential model for predicting PPV in open - pit mines, namely ICA ANN. Finally, they introduced that this model can predict PPV with high reliability, and it can be used instead of empirical models. In addition, many other studies were developed or proposed AI techniques for predicting PPV with high performance (Nguyen et al., 2019b; Shang et al., 2019; Yang et al., 2019; Zhang et al., 2019).

A review of the literature shows that AI techniques have been successfully applied in predicting PPV in open - pit mines. Nonetheless, they have not been applied anywhere. In this study, the $\mathrm{k}$ - nearest neighbors (KNN) algorithm was investigated and applied to predict PPV at the Deo Nai open - pit coal mines (Vietnam). An empirical model was also taken into account and compared with the KNN model to have a comprehensive assessment of PPV prediction.

\section{Background of $k$ - nearest neighbors algorithm}

A $k$ - nearest neighbor interpolation is a statistical tool that is used to estimate the value of an unknown or missing point based on its nearest neighbors (Peterson, 2009). The nearest neighbors are usually determined as the points with the shortest distance to the unknown point from its contiguity (Gou et al., 2019). There are several techniques used to measure the distance between the neighbors; the simplest one is the Euclidian distance function given in (1).

$$
d(x, y)=\|x-y\|=\sqrt{\sum_{i=1}^{n}\left(x_{i}-y_{i}\right)^{2}}
$$

Where $x=\left(x_{1}, x_{2}, \ldots, x_{n}\right)$ and $y=\left(y_{1}, y_{2}, \ldots, y_{n}\right)$, and $n$ is the vector size. The $k$ neighbor points that have the shortest distance to the unknown point is used to estimate its value using (2).

$$
\hat{y}_{i}=\sum_{i=1}^{n} w_{i} y_{i}
$$

Where $w_{i}$ is the weight of every single neighbor point $y_{i}$ to the query point $y$ (Härdle and Linton, 1994).

The KNN interpolation defined in (2) is the weighted average of the neighborhood. The simplest KNN model is the mean of the contiguity, which is obtained in the case of the uniform weights where all the neighbor points have the same effect on the estimation $\left(w_{i}=\frac{1}{n}\right)$. On the contrary, when the neighbor points are assumed to have a different effect on the estimation of the 
query point, different weights can be applied. The simplest weight function is given in (3).

$$
w_{i}=\frac{d_{i}}{\sum_{i=1}^{n} d_{i}}
$$

Where $d_{i}$ is the distance between the unknown point and its neighbor. The weight function must gain its maximum value at zero distance from the interpolated point, and as the distance increases the function should decrease respectively (Atkeson et al., 1997). A list of different weights can be found in (Syaliman et al., 2018; Sun et al., 2019).

The KNN estimation presented in (2) depends only on the neighbor points. Hence, it ignores the trend of the whole data set in the estimation process. In addition, it can be affected by extreme values resulting in an overestimated model.

\section{Case study}

This study was undertaken in the Deo Nai open - pit coal mine, which located in the Quang Ninh province (Vietnam), as shown in Figure 1. In this mine, blasting was selected as the main method for rock fragmentation before loading and transporting. ANFO and emulsion explosives were used in this mine, and ANFO is the primary explosive used herein. The boreholes with the diameters in the range of $105 \mathrm{~mm}$ to $250 \mathrm{~mm}$ were applied in this mine for blasting, and the millisecond - delay blasting method was applied.

For the data collection, this study collected eight parameters, including maximum explosive charge per delay $(Q)$, the hole depth $(L)$, burden $(W)$, spacing $(B)$, stemming $(L B)$, powder factor $(q)$, monitoring distance $(D)$, and PPV. Of those, the first seven parameters were used as the input parameters, and the last one (i.e., PPV) was used as the output parameter. Herein, the PPV was

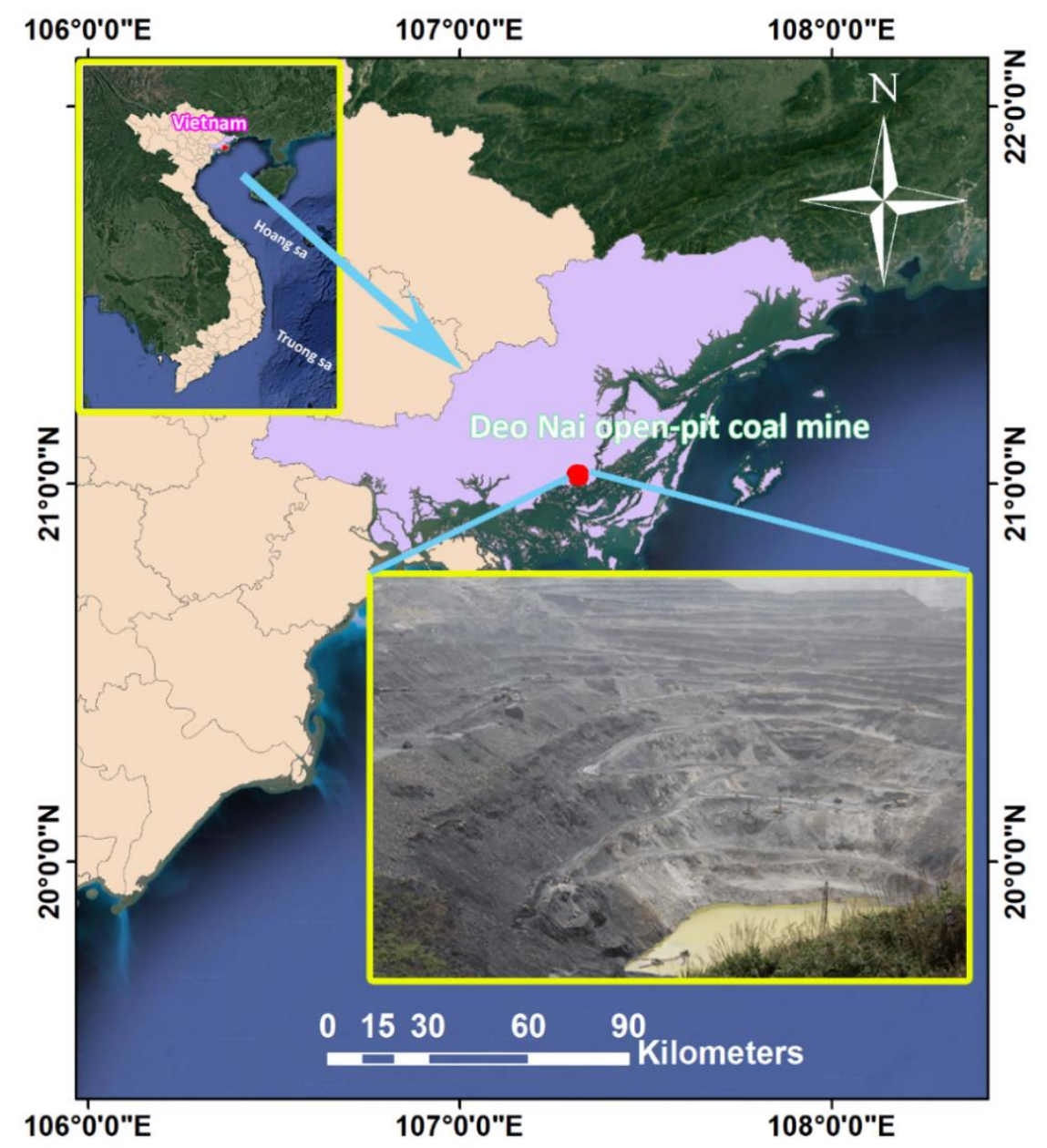

Figure 1. Location and a view of the Deo Nai open - pit coal mine (Vietnam). 
measured by the blastmate III or micromate (Instantel - Canada), $D$ was calculated based on the locations of blast sites and measurement points that were pointed by a GPS receiver. The remaining parameters were extracted from blast patterns. Finally, 194 blasting events were recorded, and the dataset was summarized in Figure 2.

\section{Development of the models}

In this section, the details of the models' development are presented. As mentioned in the introduction section, this study aims at applying the KNN algorithm for predicting PPV at the Deo Nai open - pit coal mine. Also, an empirical model was developed to compare with the KNN model.

Before developing the models, the dataset was divided into two sections: $80 \%$ of the whole dataset was used for training the models, and the remaining $20 \%$ of the dataset was used for testing

$Q$

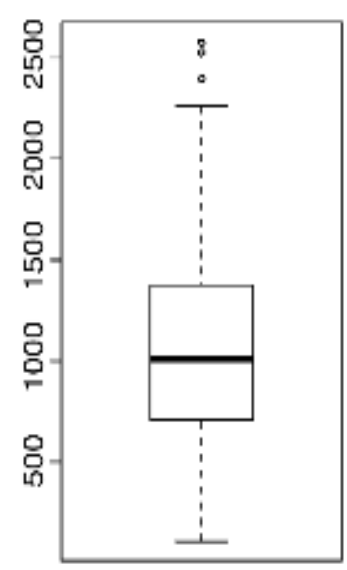

LB

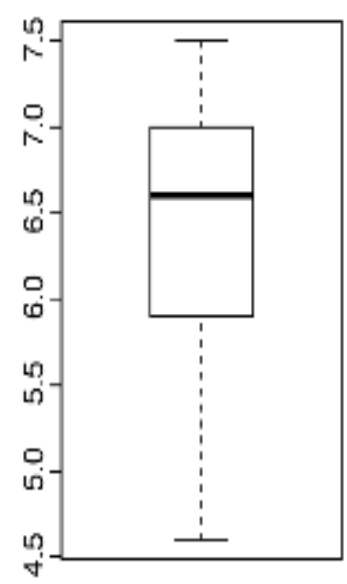

L

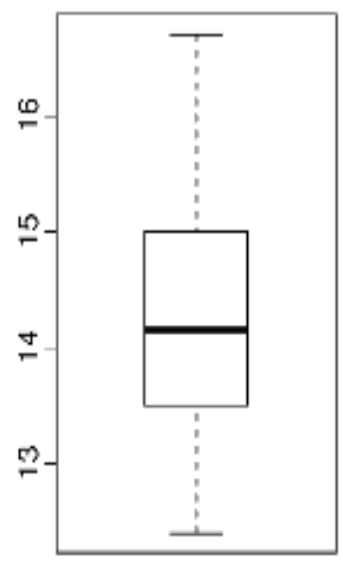

q

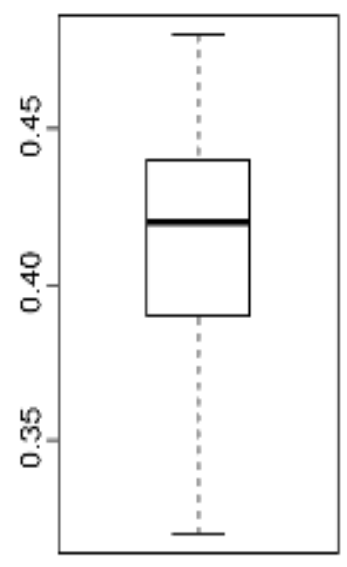

the developed models. It is worth noting that this task was performed randomly.

For the development of the KNN model, the number of " $k$ nearest neighbors" $(k)$ and their distance $(d)$ were used as the main parameter to control the accuracy of the KNN model. Also, different kernel functions were applied during training the KNN model aiming to map the dataset to higher feature space, such as inv, rectangular, triangular, triweight, biweight, cos, epanechnikov, and gaussian. In order to avoid overfitting of the KNN model, 10 - fold cross - validation technique, and the MinMax scale $[0,1]$ were applied. A trial and error procedure with the maximum neighbors in the range of 1 to 52 , their distance in the range of 0 to 3 , was applied to find out the best KNN model. Finally, one hundred KNN models were developed, as shown in Figure 3. The best KNN model was then defined with $k=35, d=$ 0.215 , and the inv kernel function (Figure 3 ).

w

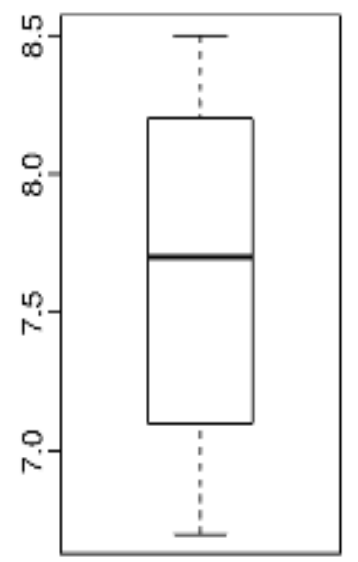

D

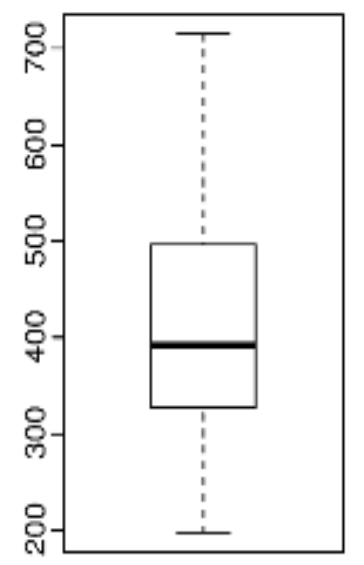

B

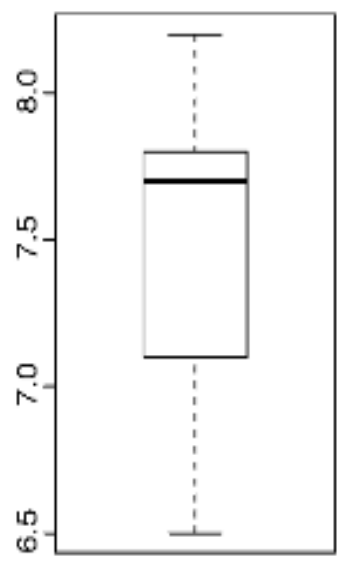

PPV

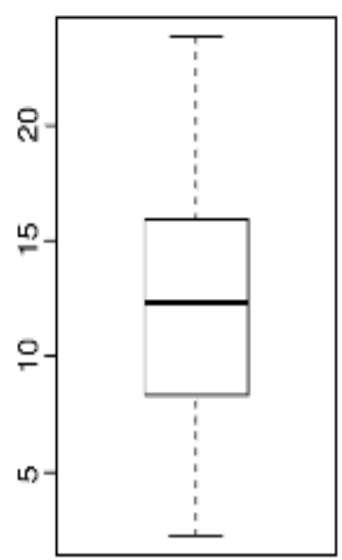

Figure 2. Summary of the dataset used in this study. 


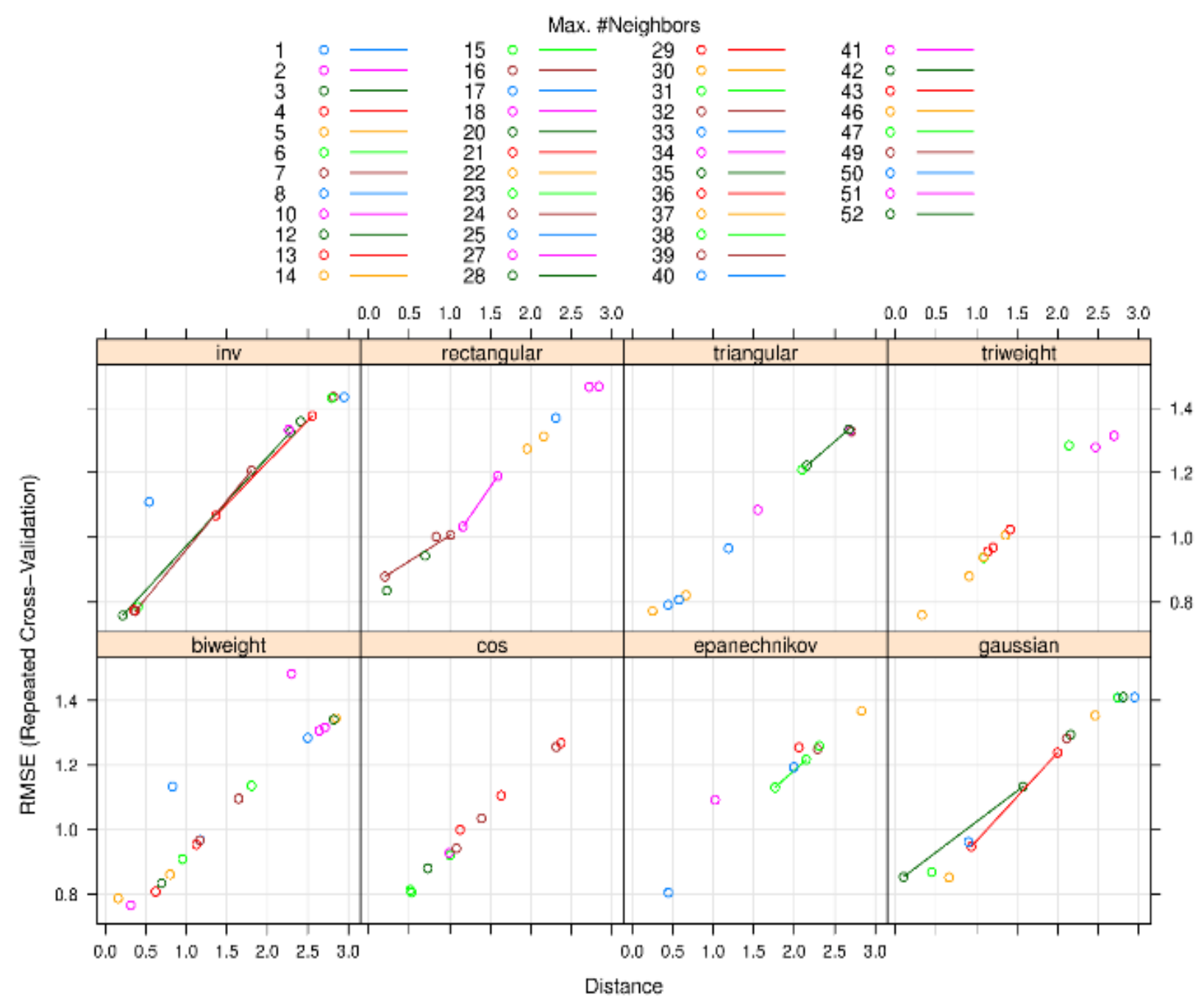

Figure 3. Performance of the KNN models with different parameters and kernel functions.

For the empirical model, the U.S Bureau of Mines (USBM) empirical equation (Duvall and Petkof, 1958) was applied for estimating PPV, as follows:

$$
\mathrm{PPV}=\lambda\left(\frac{\mathrm{Q}}{\sqrt{\mathrm{D}}}\right)^{-\alpha}
$$

where $Q$ stands for the maximum explosive charge per delay (in $\mathrm{kg}$ ); D stands for the monitoring distance (m); $\lambda$ and $\alpha$ were the site parameters and were considered using the multivariate regression analysis. Finally, the USBM empirical equation was defined as follows:

$$
\mathrm{PPV}=1.493\left(\frac{\mathrm{Q}}{\sqrt{\mathrm{D}}}\right)^{0.524}
$$

\section{Assessment of the models}

Once the KNN and empirical models were well - developed based on the training dataset, the testing dataset was used to validate the performance of the models. To evaluate the performance as well as the accuracy of the models, root - mean - squared error (RMSE), determination coefficient $\left(R^{2}\right)$ and mean absolute error (MAE) were used as the performance metrics, and they are calculated as follow:

$$
\mathrm{RMSE}=\sqrt{\frac{1}{n} \sum_{i=1}^{n}\left(y_{P P V i}-\hat{y}_{P P V i}\right)^{2}}
$$




$$
\begin{array}{r}
\mathrm{R}^{2}=1-\frac{\sum_{i=1}^{n}\left(y_{P P V i}-\hat{y}_{P P V i}\right)^{2}}{\sum_{i=1}^{n}\left(y_{P P V i}-\bar{y}_{P P V i}\right)^{2}} \\
\mathrm{R}^{2}=\frac{1}{n} \sum_{i=1}^{n}\left|y_{P P V i}-\hat{y}_{P P V i}\right|
\end{array}
$$

Where $n$ stands for a total number of observations; $\gamma_{P P V_{i}}$ is the measured PPV, $\gamma_{P P V_{i}}$ is predicted PPV, and $\gamma_{P P V_{i}}$ is the mean of $\gamma_{P P V_{i}}$. The results of the KNN and USBM empirical models are shown in Table 1.

From the results in Table 1, it can be seen that the KNN model provided much better performance than those of the USBM model with an RMSE of 0.759 and $R^{2}$ of 0.974 on the training dataset, and RMSE of 1.157 and $R^{2}$ of 0.967 on the testing dataset. In contrast, the USBM empirical model yielded a bad performance with an RMSE of 3.619; $R^{2}$ of 0.461 ; and MAE of 2.794 on the training dataset and RMSE of $4.205 ; R^{2}$ of 0.416 , and MAE of 3.361 on the testing dataset. For further assessment of the models, the chart of the correlation between measured and predicted
PPVs by the KNN and USBM empirical models was used, as shown in Figure 4.

Based on the observations in Figure 4, it is clear that the correlation between measured and predicted PPVs by the KNN model is much better than those of the USBM model. On the other hand, most of the predicted PPVs are inside of the $80 \%$ confidence level of the KNN model. Whereas, most of the predicted PPVs of the USBM are outside of the $80 \%$ confidence level. This finding indicated that the USBM empirical model is not suitable for predicting PPV in this case study. In contrast, the KNN model is a robust AI model for predicting PPV at the Deo Nai open - pit coal mine with a promising result (i.e., $R M S E=1.157, R^{2}=0.967$, and $M A E=0.602$ ).

\section{Conclusion}

Blasting is an effective method for fragmenting rock; however, its side effects are significant for the surrounding environment, especially blast - induced ground vibration. This study investigated and developed a KNN model for predicting blast - induced ground vibration in open - pit mines, and it was applied to the Deo Nai open - pit coal mine (Vietnam) as a case study.

Table 1. Results of the KNN and USBM models based on both training and testing datasets.

\begin{tabular}{|c|c|c|c|c|c|c|}
\hline \multirow{2}{*}{ Model } & \multicolumn{3}{|c|}{ Training dataset } & \multicolumn{3}{c|}{ Testing dataset } \\
\cline { 2 - 7 } & $R M S E$ & $R^{2}$ & $M A E$ & $R M S E$ & $R^{2}$ & $M A E$ \\
\hline KNN & 0.759 & 0.974 & 0.467 & 1.157 & 0.967 & 0.602 \\
\hline USBM & 3.619 & 0.461 & 2.794 & 4.205 & 0.416 & 3.361 \\
\hline
\end{tabular}
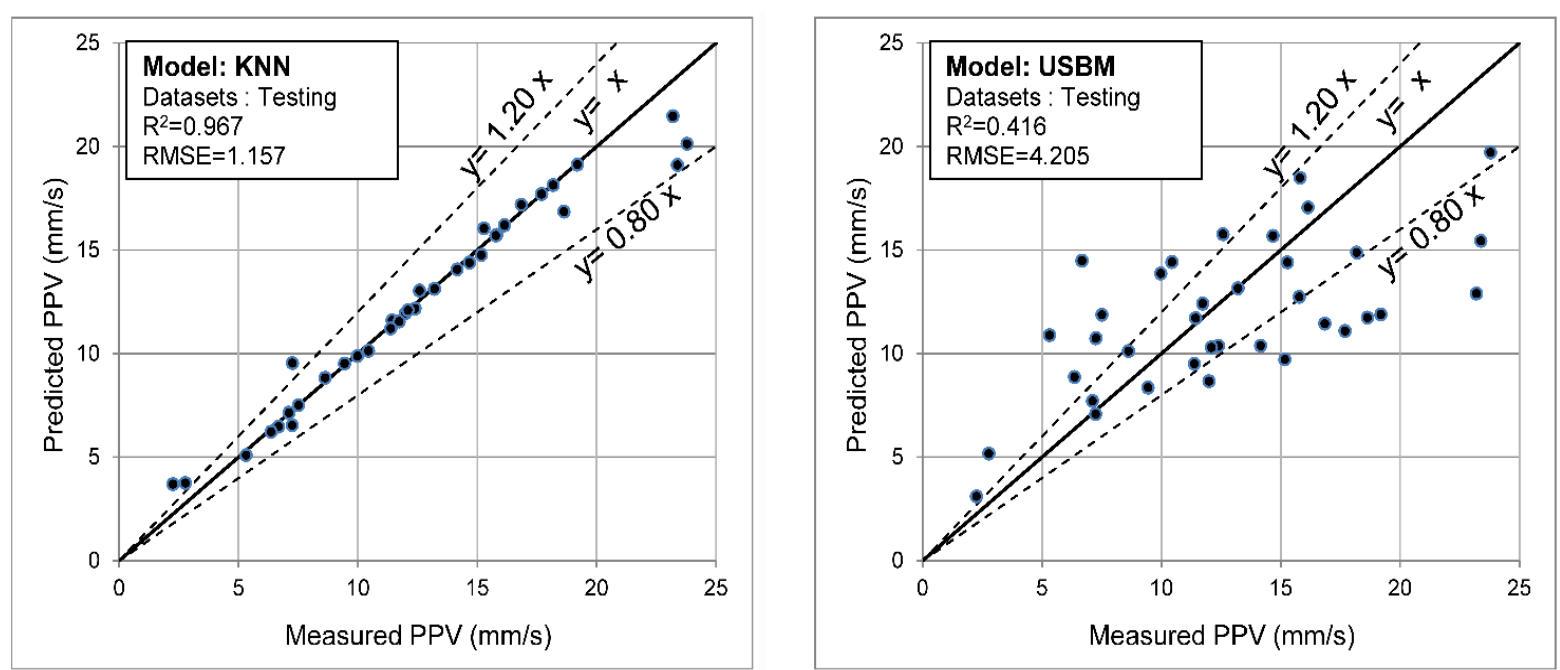

Figure 4. Correlation between measured and predicted PPVs by the KNN and USBM models. 
The results revealed that the KNN model could predict PPV with high reliability, and it can be used in practical engineering to predict and control blast - induced ground vibration. The USBM empirical model or other empirical equations should be further studied in the future to improve the accuracy in predicting PPV in open - pit mines.

\section{Acknowledgments}

This paper was supported by the Ministry of Education and Training (MOET) in Viet Nam under grant number B2020 - MDA - 16. The authors also thank the Center for Mining, Electro Mechanical research of Hanoi University of Mining and Geology (HUMG), Hanoi, Vietnam, and the research team of Innovations for Sustainable and Responsible Mining (ISRM) of HUMG.

\section{References}

Armaghani, D. J., Hajihassani, M., Mohamad, E. T., Marto, A. and Noorani, S., (2014). Blasting induced flyrock and ground vibration prediction through an expert artificial neural network based on particle swarm optimization. Arabian Journal of Geosciences, 7(12): 5383 - 5396.

Armaghani, D. J., Hasanipanah, M., Amnieh, H. B. and Mohamad, E. T., (2018). Feasibility of ICA in approximating ground vibration resulting from mine blasting. Neural Computing and Applications, 29(9): 457 - 465.

Armaghani, D. J., Momeni, E., Abad, S. V. A. N. K. and Khandelwal, M., (2015). Feasibility of ANFIS model for prediction of ground vibrations resulting from quarry blasting. Environmental Earth Sciences, 74(4): 2845 - 2860.

Atkeson, C. G., Moore, A. W. and Schaal, S., (1997). Locally weighted learning for control. Lazy learning, Springer: 75 - 113.

Bui, X. - N., Choi, Y., Atrushkevich, V., Nguyen, H., Tran, Q. - H., Long, N. Q. and Hoang, H. - T., (2020). Prediction of Blast - Induced Ground Vibration Intensity in Open - Pit Mines Using Unmanned Aerial Vehicle and a Novel Intelligence System. Natural Resources Research, 29(2): 771 - 790, DOI: 10.1007/s11053 - 019 - 09573 - 7.
Bui, X. - N., Nguyen, H., Le, H. - A., Bui, H. - B. and Do, N. - H., (2019). Prediction of Blast - induced Air Over - pressure in Open - Pit Mine: Assessment of Different Artificial Intelligence Techniques. Natural Resources Research, 29(2): 571 - 591, DOI: 10.1007/s11053 - 019 09461 - 0 .

Ding, Z., Nguyen, H., Bui, X. - N., Zhou, J. and Moayedi, H., (2019). Computational Intelligence Model for Estimating Intensity of Blast - Induced Ground Vibration in a Mine Based on Imperialist Competitive and Extreme Gradient Boosting Algorithms. Natural Resources Research, DOI: 10.1007/s11053 019 - 09548 - 8.

Duvall, W. I. and Petkof, B., (1958). Spherical propagation of explosion - generated strain pulses in rock, Bureau of Mines

Fang, Q., Nguyen, H., Bui, X. - N. and Nguyen - Thoi, T., (2019a). Prediction of Blast - Induced Ground Vibration in Open - Pit Mines Using a New Technique Based on Imperialist Competitive Algorithm and M5Rules. Natural Resources Research, 29(2): 791 - 806, DOI: $10.1007 / \mathrm{s} 11053$ - 019 - 09577 - 3.

Fang, Q., Nguyen, H., Bui, X. - N. and Tran, Q. - H., (2019b). Estimation of Blast - Induced Air Overpressure in Quarry Mines Using Cubist Based Genetic Algorithm. Natural Resources Research, 29(2): 593 - 607, DOI: 10.1007/s11053 - 019 - 09575 - 5.

Gou, J., Ma, H., Ou, W., Zeng, S., Rao, Y. and Yang, H., (2019). A generalized mean distance - based $\mathrm{k}$ - nearest neighbor classifier. Expert Systems with Applications, 115: 356 - 372.

Hajihassani, M., Armaghani, D. J., Marto, A. and Mohamad, E. T., (2015). Ground vibration prediction in quarry blasting through an artificial neural network optimized by imperialist competitive algorithm. Bulletin of Engineering Geology and the Environment, 74(3): 873 - 886.

Härdle, W. and Linton, O., (1994). Applied nonparametric methods. Handbook of econometrics, 4: 2295 - 2339.

Hasanipanah, Faradonbeh, Amnieh, Armaghani and Monjezi, (2017). Forecasting blast - 
induced ground vibration developing a CART model. Engineering with Computers, 33(2): 307 - 316.

Khandelwal, M., (2011). Blast - induced ground vibration prediction using support vector machine. Engineering with Computers, 27(3): $193-200$.

Monjezi, M., Ahmadi, M., Sheikhan, M., Bahrami, A. and Salimi, A., (2010). Predicting blast induced ground vibration using various types of neural networks. Soil Dynamics and Earthquake Engineering, 30(11): 1233 - 1236.

Monjezi, M., Hasanipanah, M. and Khandelwal, M., (2013). Evaluation and prediction of blast induced ground vibration at Shur River Dam, Iran, by artificial neural network. Neural Computing and Applications, 22(7 - 8): 1637 1643.

Nguyen, H., (2019). Support vector regression approach with different kernel functions for predicting blast - induced ground vibration: a case study in an open - pit coal mine of Vietnam. SN Applied Sciences, 1(4): 283.

Nguyen, H. and Bui, X. - N., (2019). Predicting Blast - Induced Air Overpressure: A Robust Artificial Intelligence System Based on Artificial Neural Networks and Random Forest. Natural Resources Research, 28(3): 893 - 907, DOI: 10.1007/s11053 - 018 - 9424 - 1.

Nguyen, H., Bui, X. - N., Choi, Y., Lee, C. W. and Armaghani, D. J., (2020). A Novel Combination of Whale Optimization Algorithm and Support Vector Machine with Different Kernel Functions for Prediction of Blasting - Induced Fly - Rock in Quarry Mines. Natural Resources Research, DOI: 10.1007/s11053 - 020 - 09710 $-7$.

Nguyen, H., Bui, X. - N., Tran, Q. - H., Le, T. - Q. and Do, N. - H., (2019a). Evaluating and predicting blast - induced ground vibration in open - cast mine using ANN: A case study in Vietnam. $S N$ Applied Sciences, 1(1): 125.
Nguyen, H., Drebenstedt, C., Bui, X. - N. and Bui, D. T., (2019b). Prediction of blast - induced ground vibration in an open - pit mine by a novel hybrid model based on clustering and artificial neural network. Natural Resources Research, 29(2): 691 - 709.

Peterson, L. E., (2009). K - nearest neighbor. Scholarpedia, 4(2): 1883.

Shang, Y., Nguyen, H., Bui, X. - N., Tran, Q. - H. and Moayedi, H., (2019). A Novel Artificial Intelligence Approach to Predict Blast Induced Ground Vibration in Open - Pit Mines Based on the Firefly Algorithm and Artificial Neural Network. Natural Resources Research, 29(2): 723 - 737, DOI: 10.1007/s11053 - 019 $09503-7$.

Sun, J., Bo, Y., Luo, J. and Yang, J., (2019). Application of the $\mathrm{K}$ Nearest Neighbor Algorithm Based on Scaling Weight in Intelligent Attendance System. 2019 11th International Conference on Measuring Technology and Mechatronics Automation (ICMTMA), IEEE.

Syaliman, K., Nababan, E. and Sitompul, O., (2018). Improving the accuracy of $\mathrm{k}$ - nearest neighbor using local mean based and distance weight. Journal of Physics: Conference Series.

Yang, H., Hasanipanah, M., Tahir, M. M. and Bui, D. T., (2019). Intelligent Prediction of Blasting Induced Ground Vibration Using ANFIS Optimized by GA and PSO. Natural Resources Research, 29(2): 739 - 750, DOI: $10.1007 / \mathrm{s} 11053$ - 019 - 09515 - 3.

Zhang, X., Nguyen, H., Bui, X. - N., Tran, Q. - H., Nguyen, D. - A., Bui, D. T. and Moayedi, H., (2019). Novel Soft Computing Model for Predicting Blast - Induced Ground Vibration in Open - Pit Mines Based on Particle Swarm Optimization and XGBoost. Natural Resources Research, 29(2): 711 - 721, DOI: $10.1007 / \mathrm{s} 11053$ - 019 - 09492 - 7. 